\title{
TOEPLITZ OPERATORS ON ABSTRACT HARDY SPACES
}

\author{
by R. C. SMITH
}

(Received 4 June, 1986)

Introduction. In [10], C. Sundberg uses a clever argument involving an idea of Davie and Jewell [13] to prove an isomorphism theorem for a very general class of operators. A related spectral inclusion theorem is an immediate consequence of the proof of this result, as Sundberg points out. He goes on to list several well known examples that are applications of his main result and remarks that the proof of the McDonald-Sundberg theorem (c.f. [9]) can now be considerably simplified. The purpose of this note is to give further evidence of the utility of the criterion established in [10]. Here and throughout $X$ denotes a compact Hausdorff space and $A$ is a function algebra on $X$. The Shilov boundary of $A$ is the minimal closed subset $\partial(A)$ of $X$ with the property that

$$
\|f\|_{\infty}=\sup \{|f(x)|: x \in \partial(A)\} \text { for all } f \in A .
$$

Given a positive (regular Borel) measure $\mu$ on $X$, the abstract Hardy space $H^{2}(\mu)$ is the $L^{2}(\mu)$-closure of $A$. For $f \in L^{\infty}(\mu)$, we define the multiplication operator $M_{f}$ on $L^{2}(\mu)$ by

$$
M_{f} g=f g, \text { for } g \in L^{2}(\mu)
$$

and we define the Toeplitz operator $T_{f}$ on $H^{2}(\mu)$ by

$$
T_{f} g=P(f g), \text { for } g \in H^{2}(\mu),
$$

where $P: L^{2}(\mu) \rightarrow H^{2}(\mu)$ denotes the orthogonal projection.

Let $\mathscr{T}$ denote the $C^{*}$-algebra of bounded operators on $H^{2}(\mu)$ generated by $\left\{T_{f}: f \in C(X)\right\}$ and let $\mathscr{C}$ denote the commutator ideal of $\mathscr{T}$; that is, $\mathscr{C}$ is the ideal generated by $\left\{T_{f} T_{g}-T_{g} T_{f}: f, g \in C(X)\right\}$. Now $\mathscr{T} / \mathscr{C}$ is a commutative $C^{*}$-algebra and it is of interest to obtain a description of $\mathscr{T} / \mathscr{C}$ in terms of continuous functions on $X$. If $X$ is the closed unit disk in the complex plane, $A$ is the disk algebra, and $\mu$ is Lebesgue measure on $\partial X$, a theorem of L. A. Coburn (see Chapter 7 of [4]) asserts that $\mathscr{T} / \mathscr{C}=C(\partial X)$

In general, the recent result of $C$. Sundberg [10] tells us that $\mathscr{T} / \mathscr{C}=C(E)$ for the closed set

$E=\left\{x \in X: f \in C(X)\right.$ with $f(x)=0$ implies $M_{f}$ is not bounded below on $\left.H^{2}(\mu)\right\}$.

We can use $\mathscr{C}$ in place of the semicommutator ideal because the two ideals are the same in our context. As a consequence of his proof, Sundberg obtains the inclusion $f(E) \subset \sigma\left(T_{f}\right)$.

Main result. Our goal is to further describe this set $E$, but first we need a notion from the theory of function algebras. A closed set $F$ in $X$ is said to be a peak set for $A$ if there is a function $f \in A$ with $f=1$ on $F$ and $|f|<1$ off $F$. A point $x \in X$ is a weak peak

Glasgow Math. J. 30 (1988) 129-131. 
point for $A$ if $\{x\}$ is an intersection of peak sets. It is not hard to see that the weak peak points for $A$ are dense in $\partial(A)$. The reader is referred to [5] as a general reference for function algebras.

THEOREM. If $\operatorname{supp} \mu \subset \partial(A)$, then $E=\operatorname{supp} \mu$ (i.e., $\mathscr{T} / \mathscr{C}=C(\operatorname{supp} \mu)$ ). In general, $\partial(A) \cap \operatorname{supp} \mu \subset E$.

Proof. First of all, it is clear that $E \subset \operatorname{supp} \mu$. Thus we need only prove the containment $\partial(A) \cap \operatorname{supp} \mu \subset E$. Towards this end, suppose $f$ is a continuous function that vanishes at $y \in \partial(A) \cap \operatorname{supp} \mu$. For convenience, let $\|f\|_{\infty}=1$. For any $\varepsilon>0$, there is an open set $V$ containing $y$ such that $|f|<\varepsilon$ on $V$. But $V$ contains a weak peak point $x$ for $A$, and so there exists $h \in A$ with $h(x)=\|h\|_{\infty}=1$ and $|h|<1$ on $X \backslash V$. Choose $0<\alpha<\beta<1$ such that $|h| \leqq \alpha$ on $X \backslash V$ and put $S=\{t \in X:|1-h(t)| \leqq 1-\beta\}$. By Runge's theorem, there is a sequence $\left\{p_{n}\right\}$ of polynomials such that $p_{n} \rightarrow 0$ uniformly on $\{z:|z| \leqq \alpha\}$ and $p_{n} \rightarrow 2 \mu(S)^{-1 / 2}$ uniformly on $\{z:|1-z| \leqq 1-\beta\}$. Because $p_{n}(h) \in A$, there is a function $g \in A$ with $\int|g|^{2} d \mu \geqq 1$ and with $|g|<\varepsilon$ on $X \backslash V$. Now

$$
\begin{aligned}
\int|f g|^{2} d \mu & \leqq \varepsilon^{2} \int_{V}|g|^{2} d \mu+\varepsilon^{2} \int_{X V V}|f|^{2} d \mu \\
& \leqq \varepsilon^{2} \int|g|^{2} d \mu+\varepsilon^{2} \leqq 2 \varepsilon^{2} \int|g|^{2} d \mu .
\end{aligned}
$$

Thus $\left\|M_{f} g\right\|_{2} \leqq 2^{1 / 2} \varepsilon\|g\|_{2}$, whereby $y \in E$.

Corollary. For $f \in C(X)$, we have $f(\partial(A) \cap \operatorname{supp} \mu) \subset \sigma\left(T_{f}\right)$.

Remarks. If $A=H^{\infty}$, the bounded analytic functions on the open unit disk in the complex plane and if $\mu$ is the natural lift to $\partial\left(H^{\infty}\right)$ of Lebesgue measure $m$ on the unit circle, we obtain $f\left(\partial\left(H^{\infty}\right)\right) \subset \sigma\left(T_{f}\right)$. Now $\partial\left(H^{\infty}\right)$ can be identified with the maximal ideal space of $L^{\infty}(m)$ and $L^{\infty}(m)=C\left(\partial\left(H^{\infty}\right)\right)$ under this identification. Letting $\mathscr{R}(f)$ denote the essential range of $f$ in $L^{\infty}(m)$, and taking the Toeplitz operator $T_{f}$ acting on the classical Hardy space, we obtain the well known result of R. G. Douglas (see Chapter 7 of [4]) that $\mathscr{R}(f) \subset \sigma\left(T_{f}\right)$. In view of the previously mentioned theorem of L. A. Coburn, it seems natural to ask if similar results hold for algebras $A$ that lie between the disk algebra and $H^{\infty}$. This was the question that originally motivated this research. Of course, we were able to obtain more.

Theorem 2 of [7] asserts that the first statement of our main result holds for function algebras that approximate in modulus, while Theorem 2 of [8] is a version of our main result for hypo-Dirichlet algebras but with the restriction that the measure $\mu$ be a logmodular measure for a multiplicative linear functional on $A$. Given a compact set $X$ in the complex plane, let

$$
A=A(X)=\{f \in C(X): f \text { is analytic on the interior of } X\}
$$

and let supp $\mu \subset X$. In the case $X=\operatorname{supp} \mu$, because $\partial(A)=\partial X$, we obtain $f(\partial X) \subset \sigma\left(T_{f}\right)$ for $f \in C(X)$, generalizing certain cases of a result in [2] for Bergman spaces. 
Furthermore, an example in [2] shows that $f(X)$ neither contains $\sigma\left(T_{f}\right)$ nor is contained in $\sigma\left(T_{f}\right)$. In the case $\partial X=\operatorname{supp} \mu$, we again get $f(\partial X) \subset \sigma\left(T_{f}\right)$, generalizing results in [1] and [8] concerning Hardy spaces of finitely connected planar domains. Of course these results hold for any algebra $A$ on $X$ with $\partial(A)=\partial X$; e.g., the $T$-invariant algebras in [6]. Similar results also hold in several variables when the Shilov boundary can be identified. Finally, note that the McDonald-Sundberg theorem of (cf. [9]) shows that $\partial(A) \cap \operatorname{supp} \mu$ is not necessarily equal to $E$ even when $\partial(A) \subset E$.

The author would like to thank Sheldon Axler and Paul Bourdon for some valuable conversations regarding the work presented here.

\section{REFERENCES}

1. M. B. Abrahamse, Toeplitz operators in multiply connected domains, Amer. J. Math. 96 (1974), 261-297.

2. S. Axler, J. B. Conway and G. McDonald, Toeplitz operators on Bergman spaces, Canad. J. Math. 34 (1982), 466-483.

3. A. M. Davie and N. P. Jewell, Toeplitz operators in several complex variables, $J$. Functional Analysis 26 (1977), 356-368.

4. R. G. Douglas, Banach algebra techniques in operator theory (Academic Press, 1972).

5. T. W. Gamelin, Uniform algebras (Prentice Hall, Englewood Cliffs, New Jersey, 1969).

6. T. W. Gamelin, Rational approximation theory (UCLA Course Notes, 1975). $157-161$.

7. J. Janas, Toeplitz operators for a certain class of function algebras, Studia Math. 55 (1976), 249-254.

8. J. Janas, Toeplitz operators for hypo-Dirichlet algebras, Ann. Polon. Math. 37 (1980),

9. G. McDonald and C. Sundberg, Toeplitz operators on the disc, Indiana Univ. Math. J. 28 (1979), 595-611.

10. C. Sundberg, Exact sequences for generalized Toeplitz operators, preprint.

Department of Mathematics and Statistics

MississipPI State UNIVERSITY

MississipPI 39762

USA 